\title{
FREE COALGEBRAS IN A CATEGORY OF RINGS
}

\author{
ROBERT DAVIS
}

Abstract. Let $R$ be the category of commutative rings with unity and unity-preserving homomorphisms, and let $\Pi$ be a small algebraic theory, i.e., an algebraic theory with a rank in the sense of Linton. The category $Q$ of II-coalgebras in $R$ is the category of coproduct-preserving functors $\Pi^{*} \rightarrow \Omega$. We prove that the standard forgetful functor $U: Q \rightarrow R$ has a right adjoint $V$.

Part I. We use the following notation in addition to that of the Abstract: if $B$ is a $\Pi$-coalgebra in $R$ and $\omega: n \rightarrow 1$ in $\Pi$, we write $\omega_{B}: B \rightarrow n B$, where $n B=\otimes^{n} B$ is the $n$-fold tensor product (coproduct in $R$ ). We prove first that $V A$ exists whenever the additive group of the ring $A$ is torsion-free. Let $B$ be a $\Pi$-coalgebra and $f: B \rightarrow A$ be a ring homomorphism. Define $g_{\omega}=(a(\omega) f) \circ \omega_{B}: B \rightarrow a(\omega) A$ for each $\omega$ of arity $a(\omega)$ less than the rank of $\Pi$, and let $g: B \rightarrow \prod_{\omega}(a(\omega) A)$ be determined by $p_{\omega} \circ g=g_{\omega}$. Factor the map $g$ as follows:

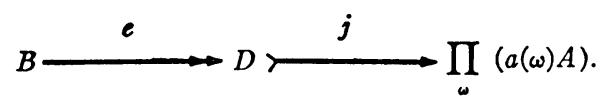

Note that $f$ factors through $e$, namely $f=p_{i d} j e$. We claim that $D$ admits a $\Pi$-coalgebra structure such that $e$ is a homomorphism. To see this, fix a map $\theta: n \rightarrow 1$ in $\Pi$ and consider the following diagram:

where:

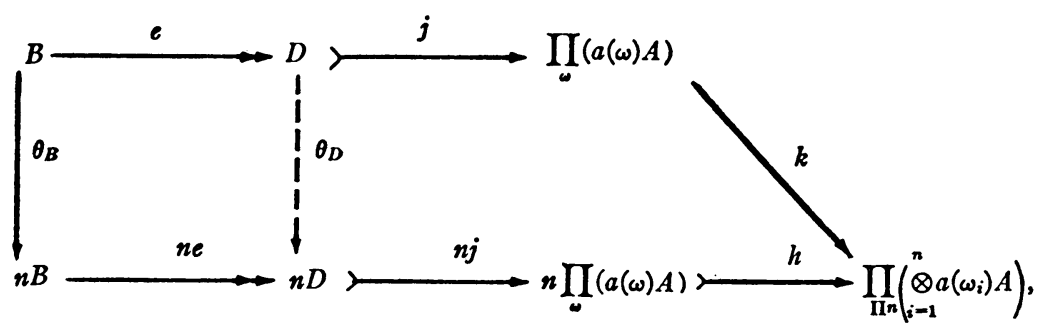

$$
\begin{aligned}
& p\left(\omega_{1}, \cdots \omega_{n}\right) \circ h=p_{\omega_{1}} \otimes \cdots \otimes p_{\omega_{n}}, \\
& \left.p\left(\omega_{1}, \cdots \omega_{n}\right) \circ k=p_{\left(\omega_{1}\right.} \otimes \cdots \otimes \omega_{n}\right) \theta_{1}
\end{aligned}
$$

Received by the editors August 7, 1969.

A MS Subject Classifications. Primary 0830, 1310; Secondary 1405.

Key Words and Phrases. Algebraic theory with a rank, right adjoint, cosolution set, tensor product of rings. 
and $n j$ and $h$ are monic since $A$ is torsion-free.

The last assertion is not obvious, so we indicate why it should be true:

Lemma. If $A$ is torsion-free, $h$ is monic.

Proof (Sketch). Note that $a(\omega) A$ is also torsion-free, so we consider instead a family $\left\{A_{\omega} \mid \omega \in \Pi\right\}$ of torsion-free rings. By passing to divisible hulls if necessary we can assume that the additive group of each $A_{\omega}$ is a rational vector space. Now, $|\Pi|$ is not finite, but if it were we could prove the lemma by selecting a basis for each $A_{\omega}$ and computing straightforwardly that any element in the intersection of the kernels of all the maps $p_{\omega_{1}} \otimes \cdots \otimes p_{\omega_{n}}$ must be zero. Thus, if $\Pi$ is replaced by any of its finite subsets, say $E$, the resulting map $h$ is monic. But $\prod A_{\omega}$ is the inverse limit of $\prod\left\{A_{\omega} \mid \omega \in E\right\}$ as $E$ varies over the finite subsets of $\Pi$. From this, it is easy to see that for the truth of the lemma, it suffices that for any integer $n$ and any directed system $A_{\delta}$ of rational vector spaces, the natural map $k: \otimes^{n}\left(\operatorname{proj} \lim A_{\delta}\right) \rightarrow \operatorname{proj} \lim \left(\otimes^{n} A_{\delta}\right)$ be monic. This does not seem to be a standard result so we indicate a proof for $n=2$. Let $A=$ proj $\lim A_{\delta}, \quad 0 \neq \alpha=\sum_{i=1}^{m}\left(a_{\delta i}\right) \otimes\left(b_{\delta i}\right) \in A \otimes A, \quad$ and suppose $k(\alpha)=0=\left(\sum a_{\delta i} \otimes b_{\delta i}\right)$. Then it is possible to find a cofinal set of indices $\delta$ and two sets $M$ and $N$ of integers between 1 and $m$ such that for each $\delta,\left\{a_{\delta i} \mid i \in M\right\}$ is a maximal independent subset of the dependent set $\left\{a_{\delta i} \mid 1 \leqq i \leqq m\right\}$ and similarly for $N$ and the $b$ 's. By definition of directed system and inverse limit it is easily seen that the expression of each $a_{\delta i}$ in terms of the independent ones is independent of $\delta$, and substitution and calculation show that the coefficients must all be zero. Thus, in each term $\left(a_{\delta i}\right) \otimes\left(b_{\delta i}\right)$, at least one factor must be zero, for otherwise the nonzero terms in some entry of $k(\alpha)$ will be independent. Hence the kernel of $k$ consists only of zero.

This completes the proof of the lemma, and we return to the diagram preceding the statement of the lemma.

The outside of this diagram commutes, so that if $b \in B, e(b)=0$, then $k j e(b)=0$ so $h(n j)(n e) \theta_{B}(b)=0$, which implies $(n e) \theta_{B}(b)=0$. Thus, a unique $\theta_{D}$ exists making the square commute; by the uniqueness, the passage $\theta \mapsto \theta_{D}$ must satisfy the (duals of the) equations of $\Pi$, so $D$ is a $\Pi$-coalgebra and $e$ is a $\Pi$-homomorphism. Thus, the set of all $\Pi$-coalgebra structures on subrings of $\prod_{\omega}(a(\omega)(A))$ forms a cosolution set for $A$. Hence, by the adjoint functor theorem, $V A$ must exist.

Part II. Tensor powers of monics need not be monic if the codomain 
is not torsion-free, so further argument is needed to establish the theorem for arbitrary rings $A$. Observe first that it suffices to prove the theorem for free theories $\Pi$, i.e., theories with no equations. For, suppose $\Pi_{1} \rightarrow \Pi$ where $\Pi_{1}$ is free and suppose that for each ring $A$ the free $\Pi_{1}$-coalgebra $V_{1} A$ exists. Let $B$ be a $\Pi$-algebra and $f: B \rightarrow A$ a ring homomorphism. Then $f$ factors through a $\Pi_{1}$-homomorphism $B \rightarrow V_{1} A$ whose image must be a $\Pi$-coalgebra. Hence the set of all $\Pi$-coalgebras contained in $V_{1} A$ is a cosolution set for $A$.

Part III. Let $\Pi$ be a free theory and $f: B \rightarrow A$ as before, except that $A$ may be any ring. Choose $\theta: n \rightarrow 1$, one of the free generators of $\Pi$, and let $\rho: \bar{A} \rightarrow A, \sigma: \bar{B} \rightarrow B$ where $\bar{A}$ and $\bar{B}$ are free commutative rings with unity. Then $V \bar{A}$ exists by Part I. Since $n \sigma$ is onto, there exists $\theta_{\bar{B}}: \bar{B} \rightarrow n \bar{B}$ such that $(n \sigma) \theta_{\bar{B}}=\theta_{B} \sigma$; in this way, $\bar{B}$ becomes a $\Pi$-coalgebra and $\sigma$ a $\Pi$-homomorphism. Since $\rho$ is onto, there exists $g: \bar{B} \rightarrow \bar{A}$ such that $\rho g=f \sigma$. Then there exist maps $\alpha: V \bar{A} \rightarrow \bar{A}, h: \bar{B} \rightarrow V \bar{A}$ such that $h$ is a $\Pi$-homomorphism and $\alpha h=g$. We obtain the following commutative diagram, where we have written $\psi$ for $\theta_{V \bar{A}}$ for brevity:

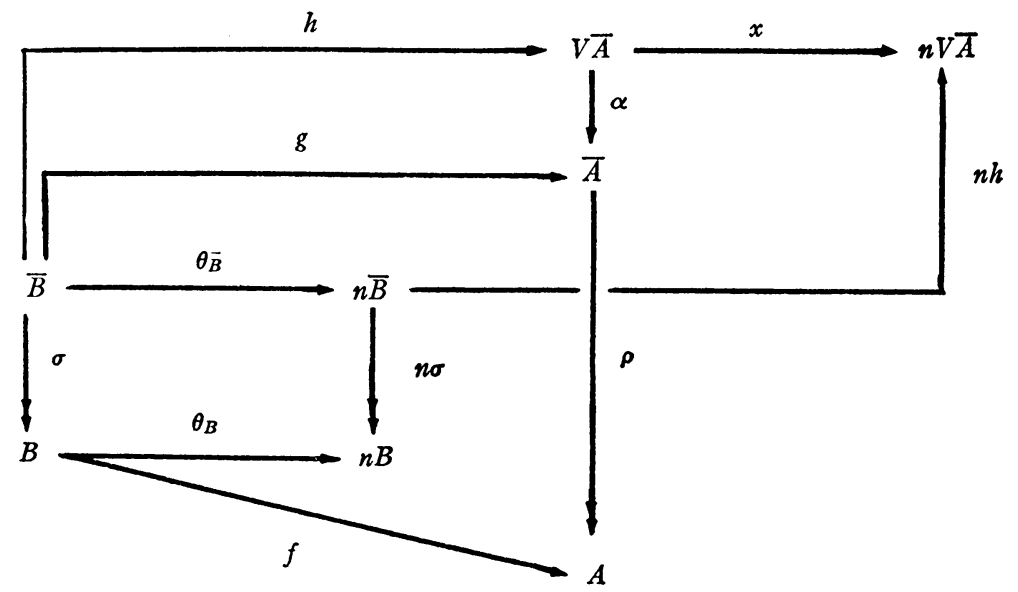

Let $\sim$ be the ring congruence on $V \bar{A}$ generated by: $h(x) \sim h(y)$ whenever $\sigma(x)=\sigma(y)$; let $k: V \bar{A} V \rightarrow \bar{A} / \sim$; and let $K$ be the kernel of $k$. Then $K$ is contained in the kernel of $\rho \alpha$, since $\sigma(x)=\sigma(y)$ implies $\rho \alpha h(x)=f \sigma(x)=f \sigma(y)=\rho \alpha h(y)$. Hence $\rho \alpha$ factors through $k$, and there is a map $V \bar{A} / K \rightarrow A$ through which $f$ factors via a map $j: B \rightarrow V \bar{A} / K$. We claim that $V \bar{A} / K$ admits a $\Pi$-coalgebra structure making $j$ a 
ח-homomorphism. By diagram chasing it is easy to see that for this it suffices that $a \in K$ implies $\psi(a)$ is in the kernel of $n k$, for each $\theta$.

Now, $a \in K$ implies $a=a_{1} s_{1}+\cdots+a_{m} s_{m}$, where $a_{i}$ is in $V \bar{A}$ and for each $s=s_{i}$ there is a sequence $x_{1}, x_{2}, \cdots, x_{p}$ where $s=h\left(x_{1}\right)$, $\sigma\left(x_{1}\right)=\sigma\left(x_{2}\right), h\left(x_{2}\right)=h\left(x_{3}\right), \cdots, \sigma\left(x_{p}\right)=0$. First, let $x \in \bar{B}$ such that $\sigma(x)=0$. Then $(n k) \psi h(x)=(n k)(n h) \theta_{\bar{B}}(x)$; but $(n \sigma) \theta_{\bar{B}}(x)=\theta_{B} \sigma(x)=0$ so $\theta_{\bar{B}}(x)$ is in the kernel of $n \sigma$. Thus $\theta_{\bar{B}}(x)$ is a sum of terms of the form $y_{1} \otimes y_{2} \otimes \cdots \otimes y_{q}$ in which at least one $y_{i}$ is in the kernel of $\sigma$. Then $(n k)(n h) \theta_{\bar{B}}(x)$ is a sum of terms of the form $k h y_{1} \otimes \cdots \otimes k h y_{q}$, which is 0 by the definition of $k$.

Thus in particular $(n k) \psi h\left(x_{p}\right)=0$. Suppose that for some integer $i$, $1<i \leqq p$, it has been shown that $(n k) \psi h\left(x_{i}\right)=0$. If $h\left(x_{i-1}\right)=h\left(x_{i}\right)$, then at once $(n k) \psi h\left(x_{i-1}\right)=0$. If $\sigma\left(x_{i-1}\right)=\sigma\left(x_{i}\right)$, then $\sigma\left(x_{i-1}-x_{i}\right)=0$ so we have

$$
(n k) \psi h\left(x_{i-1}\right)=(n k) \psi h\left(x_{i-1}-x_{i}\right)+(n k) \psi h\left(x_{i}\right)=0+0=0 .
$$

By induction, we conclude that $(n k) \psi(s)=(n k) \psi h\left(x_{1}\right)=0$, so $(n k) \psi(a)$ $=0$. This yields the desired conclusion about $V \bar{A} / K$. Hence the set of all quotients of $V \bar{A}$ is a cosolution set for $A$, and we conclude that $V A$ exists for all rings $A$.

REMARK. It is unknown whether this theorem holds for arbitrary algebraic categories as well as $R$.

Application. By [2], $R^{o p}$ is isomorphic to a category $Q \mathcal{F}$ of affine schemes. If $\Pi$ has a rank, $Q F^{\Pi} \rightarrow Q F$ has a left adjoint and is in fact triplable. Thus $Q F$ possesses free groups, free abelian groups, free $K$-algebras, etc.

\section{REFERENCES}

1. F. E. J. Linton, Some aspects of equational categories. Proc. Conf. Categorical Algebra (La Jolla, Calif., 1965), Springer-Verlag, Berlin and New York, 1966, pp. 84-94. MR 35 \#233.

2. I. G. MacDonald, Algebraic geometry, introduction to schemes, Benjamin, New York, 1968.

Southern Methodist University, Dalias, Texas 75222 